\section{COM701 IN COMBINATION WITH BMS-986207 (ANTI- TIGIT ANTIBODY) AND NIVOLUMAB - PRELIMINARY RESULTS OF SAFETY, TOLERABILITY AND PHARMACOKINETICS IN PATIENTS WITH ADVANCED SOLID TUMORS (NCT04570839)}

${ }^{1}$ Ecaterina Dumbrava*, ${ }^{2}$ Manish Sharma, ${ }^{3}$ Gini Fleming, ${ }^{4}$ Kyriakos Papadopoulos, ${ }^{5}$ Ryan Sullivan, ${ }^{6}$ Daniel Vaena, ${ }^{4}$ Amita Patnaik, ${ }^{6}$ Adam ElNaggar, ${ }^{7}$ Adeboye Adewoye, ${ }^{7}$ Robina Smith, ${ }^{7}$ Pierre Ferré, ${ }^{7}$ Inbal Barbiro, ${ }^{8}$ Emerson Lim, ${ }^{4}$ Drew Rasco. ${ }^{1}$ MDACC., Houston, TX, USA; ${ }^{2}$ START - Midwest., Grand Rapids, MI, USA; ${ }^{3}$ University of Chicago., Chicago, IL, USA; ${ }^{4}$ START - San Antonio., San Antonio, TX, USA; ${ }^{5}$ Massachusetts General Hospital., Boston, MA, USA; ${ }^{6}$ West Cancer Center and Rsrch Institute., Memphis, TN, USA; ${ }^{7}$ Compugen USA Inc., San Francisco, USA; ${ }^{8}$ Columbia University Cancer Center., New York City, NY, USA

Background COM701, a novel first-in-class immune checkpoint inhibitor (ICI) binds to poliovirus receptor related immunoglobulin domain containing (PVRIG) leading to enhanced activation of $\mathrm{T}$ and NK-cells. COM701 in combination with nivolumab has a favorable safety profile, is well tolerated and demonstrates antitumor activity. ${ }^{1}$ We hypothesized that the addition of BMS-986207 as a triplet thereby inhibiting the DNAM axis will have an acceptable safety/tolerability profile. We present preliminary results on safety/tolerability and pharmacokinetics (PK) parameters.

Methods Using an accelerated titration and $3+3$ study design we enrolled 14 patients (pts) with advanced solid tumors. Doses of COM701 were $0.3,1,3,10$ or $20[\mathrm{mg} / \mathrm{kg}$ IV Q4 wks]; in combination with nivolumab and BMS-986207 (both $480 \mathrm{mg}$ IV Q4 wks). Key objectives were to evaluate the safety and tolerability, to determine the recommended dose for expansion (RDFE) and to characterize preliminary pharmacokinetic parameters. Key inclusion criteria: Age $\geq 18$ yrs, histologically confirmed locally advanced or metastatic solid malignancy and has exhausted all available standard treatments. Key exclusion criteria: history of immune-related toxicities on prior immunotherapy treatment leading to discontinuation.

Results In the safety population [N=14], 12 pts reported treatment emergent adverse events (TEAEs). The most frequent TEAES $[\geq 3 \mathrm{pts}]$ were fatigue $5 \mathrm{pts}(36 \%)$, pyrexia 3 pts $(21 \%)$, vomiting 3 pts $(21 \%)$. No DLTs were reported in any of the dose levels. The most frequent tumor types enrolled: CRC $(\mathrm{n}=3)$, and prostate, melanoma and OVCA/primary peritoneal cancer $(\mathrm{n}=2$ each). Median number of prior therapies was 10 (range 1-19). Four pts had received prior immunotherapy. Serious adverse events $[\geq 2$ pts] were 2 pts (14\%) with G3 abdominal pain, 2 pts $(14 \%)$ with vomiting (1pt with G1/2 vomiting, 1 pt with G3 vomiting) all assessed by the investigator as unrelated to study drug. Preliminary PK profiles of COM701 were generally dose proportional.

Conclusions COM701 in combination with BMS-986207 and nivolumab demonstrates a favorable safety, tolerability and PK profiles. COM701 $20 \mathrm{mg} / \mathrm{kg}$ has been selected as the RDFE in combination with BMS-986207 and nivolumab (both 480 mg) all administered IV Q4 wks. The expansion cohorts are enrolling pts with platinum resistant ovarian cancer and endometrial cancer. Data cutoff 28 Jun 2021.

Acknowledgements This study is in collaboration with Bristol Myers Squibb.

Trial Registration NCT04570839

\section{REFERENCES}

1. Vaena, DA, Fleming GF et al. COM701 with or without nivolumab: Results of an ongoing phase 1 study of safety, tolerability and preliminary antitumor activity in patients with advanced solid malignancies (NCT03667716). J Clin Oncol 2021;39: (suppl 15; abstr 2504)

Ethics Approval The study obtained ethics approval form all the participating sites. All study participants gave informed consent before taking part.- 0002: START2020.15- 0003: 20210109- 0005: IRB20-1549- 0006: 21-060- 0007: IRBAAAT4904- 0012: 2020-0755- 0013: STMW2020.16- 0015: 20210109

http://dx.doi.org/10.1136/jitc-2021-SITC2021.478 